\title{
KANTOROVICH TYPE INEQUALITIES FOR ORDERED LINEAR SPACES*
}

\author{
MAREK NIEZGODA ${ }^{\dagger}$
}

Abstract. In this paper Kantorovich type inequalities are derived for linear spaces endowed with bilinear operations $\circ_{1}$ and $\circ_{2}$. Sufficient conditions are found for vector-valued maps $\Phi$ and $\Psi$ and vectors $x$ and $y$ under which the inequality

$$
\Phi(x) \circ_{2} \Phi(y) \leq \frac{C+c}{2 \sqrt{C c}} \Psi\left(x \circ_{1} y\right)
$$

is satisfied. Complementary inequalities are also given. Some results of Dragomir [J. Inequal. Pure Appl. Math., 5 (3), Art. 76, 2004] and Bourin [Linear Algebra Appl., 416:890-907, 2006] are generalized. The inequalities are applied to $C^{*}$-algebras and unital positive maps.

Key words. Kantorovich type inequality, Linear space, Bilinear operation, Preorder, $C^{*}$ algebra, Unital positive map, Matrix.

AMS subject classifications. 06F20, 15A45, 15A42, 15A48.

1. Introduction. Let $A$ be an $n \times n$ positive definite matrix such that $0<$ $m I_{n} \leq A \leq M I_{n}$ for some scalars $0<m<M$. The Kantorovich inequality asserts that (cf. [16, pp. 89-90], [20, p. 28])

$$
z^{*} A z \cdot z^{*} A^{-1} z \leq \frac{(M+m)^{2}}{4 M m}\left(z^{*} z\right)^{2},
$$

where $z \in \mathbb{C}^{n}$ is a column vector and ${ }^{*}$ means conjugate transpose. The constant $\kappa=\frac{(M+m)^{2}}{4 M m}$ is called Kantorovich constant [21, p. 688]. Note that $\sqrt{\kappa}=\frac{M+m}{2 \sqrt{M m}}$ is the ratio of the arithmetic to geometric mean of $M$ and $m$.

Let $V$ be a linear space over $\mathbb{C}$ or $\mathbb{R}$ equipped with inner product $\langle\cdot, \cdot\rangle$ and norm $\|\cdot\|=\langle\cdot, \cdot\rangle^{1 / 2}$. Dragomir [11, Theorem 2.2] proved the following Kantorovich type inequality:

$$
\|x\|\|y\| \leq \frac{|C+c|}{2 \sqrt{\operatorname{Re}(C \bar{c})}}|\langle x, y\rangle| \text { for } x, y \in V,
$$

provided scalars $c, C$ satisfy $\operatorname{Re}(C \bar{c})>0$ and

$$
0 \leq \operatorname{Re}\langle x-c y, C y-x\rangle
$$

${ }^{*}$ Received by the editors June 28, 2009. Accepted for publication February 14, 2010. Handling Editor: Miroslav Fiedler.

$\dagger$ Department of Applied Mathematics and Computer Science, University of Life Sciences in Lublin, Akademicka 13, 20-950 Lublin, Poland (marek.niezgoda@up.lublin.pl). 
(cf. [12, Theorem 1]). As observed in [12, p. 225], (1.2) generalizes Pólya-Szegö, Greub-Reinboldt and Cassels inequalities.

Inequality (1.2) is a reverse of Schwarz's inequality

$$
|\langle x, y\rangle| \leq\|x\|\|y\| \text { for } x, y \in V \text {. }
$$

A consequence of (1.4) and (1.2) is the following result of Bourin [5, Theorem 2.9]:

$$
\sum_{j=1}^{n} a_{[j]} b_{[j]} \leq \frac{M+m}{2 \sqrt{M m}} \sum_{j=1}^{n} a_{j} b_{j},
$$

where $a=\left(a_{1}, \ldots, a_{n}\right)$ and $b=\left(b_{1}, \ldots, b_{n}\right)$ are $n$-tuples of positive numbers with $0<m \leq \frac{a_{j}}{b_{j}} \leq M, j=1, \ldots, n$, and, in addition, $a_{[1]} \geq \ldots \geq a_{[n]}$ and $b_{[1]} \geq \ldots \geq b_{[n]}$ are the entries of $a$ and $b$, respectively, arranged in nonicreasing order.

For other Kantorovich type inequalities, the reader is referred to $[2,5,6,7,16$, $18,20,21]$.

In this paper we study Kantorovich type inequalities in the framework of linear spaces equipped with binary operations $\circ_{1}$ and $\circ_{2}$. We provide conditions on two (vector-valued) maps $\Phi$ and $\Psi$ and vectors $x$ and $y$ implying the validity of the inequality

$$
\Phi(x) \circ_{2} \Phi(y) \leq \frac{C+c}{2 \sqrt{C c}} \Psi\left(x \circ_{1} y\right) .
$$

Complementary inequalities are also derived.

2. Results. Throughout this paper, unless otherwise stated, for $i=1,2$,

$$
V_{i} \text { and } X_{i} \text { are linear spaces over } \mathbb{F}=\mathbb{C} \text { or } \mathbb{R}
$$

and

$$
\circ_{i}: V_{i} \times V_{i} \rightarrow X_{i} \text { is an } \mathbb{F} \text {-bilinear binary operation. }
$$

For example, $o_{i}$ can be interpret as a real inner product if $X_{i}=\mathbb{R}$, or as an algebra multiplication if $V_{i}=X_{i}$ is a distributive algebra.

In addition, we assume that $L_{i} \subset X_{i}$ is a convex cone inducing cone preorder $\leq_{i}$ on $X_{i}$ by

$$
y \leq_{i} x \text { iff } x-y \in L_{i}
$$

We also assume that

$$
0 \leq_{i} x \circ_{i} x, \quad \text { i.e., } x^{2}=x \circ_{i} x \in L_{i}, \quad \text { for } x \in V_{i} \text {. }
$$


We denote

$$
\operatorname{Sym}(u, w)=\frac{1}{2}\left(u \circ_{2} w+w \circ_{2} u\right) \text { for } u, w \in V_{2} \text {. }
$$

The following theorem is inspired by [11, Theorem 2.2] (cf. [12, Theorem 1]).

THEOREM 2.1. Under the above notation and assumptions, let $\Phi: \mathcal{A} \rightarrow V_{2}$ and $\Psi: \mathcal{B} \rightarrow X_{2}$ be maps, where $\mathcal{A} \subset V_{1}$ and $\mathcal{B} \subset X_{1}$ are nonempty sets. Let $x, y \in \mathcal{A}$ and $C, c \in \mathbb{F}$ with $C c>0$ and $C+c>0$ be such that

(i)

$$
0 \leq_{1}(x-c y) \circ_{1}(C y-x),
$$

(ii) $x \circ_{1} y=y \circ_{1} x$,

(iii) $L_{1} \subset \mathcal{B}$ and $\alpha x \circ_{1} y \in \mathcal{B}$ for $\alpha \in\{1, C+c\}$.

Assume that

$$
\begin{gathered}
\Phi(v) \circ_{2} \Phi(v) \leq_{2} \Psi\left(v \circ_{1} v\right) \quad \text { for } v \in\{x, y\}, \\
b \leq_{1} a \text { implies } \Psi(b) \leq_{2} \Psi(a) \text { for } a, b \in L_{1}, \\
\Psi(\alpha a)=\alpha \Psi(a) \text { for } \alpha=C+c \text { and } a=x \circ_{1} y, \\
\Psi\left(x \circ_{1} x\right)+\alpha \Psi\left(y \circ_{1} y\right) \leq_{2} \Psi\left(x \circ_{1} x+\alpha y \circ_{1} y\right) \text { for } \alpha=C c .
\end{gathered}
$$

Then the following Kantorovich type inequality holds:

$$
\operatorname{Sym}[\Phi(x), \Phi(y)] \leq_{2} \frac{C+c}{2 \sqrt{C c}} \Psi\left(x \circ_{1} y\right) .
$$

In particular, if $\Phi(x)$ and $\Phi(y)$ commute with respect to $\circ_{2}$, then

$$
\Phi(x) \circ_{2} \Phi(y) \leq_{2} \frac{C+c}{2 \sqrt{C c}} \Psi\left(x \circ_{1} y\right) .
$$

REMARK 2.2. In some cases Theorem 2.1 can be simplified.

(a). If $\Psi$ is linear then conditions (2.6)-(2.7) hold automatically and are superfluous in the statement of Theorem 2.1. If in addition $\Psi$ is positive (i.e. $\Psi\left(L_{1}\right) \subset L_{2}$ ) then (2.5) can be dropped out. 
(b). If $\Phi=\Psi$ then condition (2.4) represents a Kadison type inequality (see (2.19)). On the other hand, if $\Phi(x)=\left[\Psi\left(x^{2}\right)\right]^{1 / 2}$ then (2.4) holds automatically (cf. Corollary 2.5 and Theorem 2.7, part II).

(c). Condition (2.4) is necessary for (2.8) and (2.9) to hold. In fact, if $x=y$ then (2.3) is met for $c=C=1$. In this case, each of (2.8) and (2.9) reduces to (2.4).

Proof of Theorem 2.1. Since the operation $\circ_{1}$ is bilinear, (2.3) gives

$$
0 \leq_{1} C x \circ_{1} y-x \circ_{1} x-C c y \circ_{1} y+c y \circ_{1} x,
$$

which is equivalent to

$$
x \circ_{1} x+C c y \circ_{1} y \leq_{1} C x \circ_{1} y+c y \circ_{1} x,
$$

because $\leq_{1}$ is a cone preorder. Now, (ii) implies

$$
x \circ_{1} x+C c y \circ_{1} y \leq_{1}(C+c) x \circ_{1} y .
$$

By (2.1), $x \circ_{1} x+C c y \circ_{1} y \in L_{1}$, because $C c>0$ and $L_{1}$ is a convex cone. Therefore (2.10) yields $(C+c) x \circ_{1} y \in L_{1}$. Using (2.7), (2.10), (2.5) and (2.6), we derive

$$
\Psi\left(x \circ_{1} x\right)+C c \Psi\left(y \circ_{1} y\right) \leq_{2} \Psi\left(x \circ_{1} x+C c y \circ_{1} y\right) \leq_{2}(C+c) \Psi\left(x \circ_{1} y\right) .
$$

Consequently, by (2.4), we obtain

$$
\Phi(x) \circ_{2} \Phi(x)+C c \Phi(y) \circ_{2} \Phi(y) \leq_{2}(C+c) \Psi\left(x \circ_{1} y\right) .
$$

Hence, by $C c>0$,

$$
\frac{1}{\sqrt{C c}} \Phi(x) \circ_{2} \Phi(x)+\sqrt{C c} \Phi(y) \circ_{2} \Phi(y) \leq_{2} \frac{C+c}{\sqrt{C c}} \Psi\left(x \circ_{1} y\right) .
$$

On the other hand, by (2.1),

$$
0 \leq_{2}\left(\frac{1}{\sqrt[4]{C c}} \Phi(x)-\sqrt[4]{C c} \Phi(y)\right) \circ_{2}\left(\frac{1}{\sqrt[4]{C c}} \Phi(x)-\sqrt[4]{C c} \Phi(y)\right) .
$$

In consequence, by the bilinearity of $\circ_{2}$,

$$
0 \leq_{2} \frac{1}{\sqrt{C c}} \Phi(x) \circ_{2} \Phi(x)-\Phi(x) \circ_{2} \Phi(y)-\Phi(y) \circ_{2} \Phi(x)+\sqrt{C c} \Phi(y) \circ_{2} \Phi(y) .
$$

Hence

$$
\Phi(x) \circ_{2} \Phi(y)+\Phi(y) \circ_{2} \Phi(x) \leq_{2} \frac{1}{\sqrt{C c}} \Phi(x) \circ_{2} \Phi(x)+\sqrt{C c} \Phi(y) \circ_{2} \Phi(y),
$$


because $\leq_{2}$ is induced by a convex cone. Simultaneously, by (2.2),

$$
2 \operatorname{Sym}[\Phi(x), \Phi(y)]=\Phi(x) \circ_{2} \Phi(y)+\Phi(y) \circ_{2} \Phi(x) .
$$

Therefore we get

$$
2 \operatorname{Sym}[\Phi(x), \Phi(y)] \leq_{2} \frac{1}{\sqrt{C c}} \Phi(x) \circ_{2} \Phi(x)+\sqrt{C c} \Phi(y) \circ_{2} \Phi(y) .
$$

Combining (2.12) and (2.13), we obtain the required inequality (2.8).

REMARK 2.3. Let $H$ be a real linear space with an inner product $\langle\cdot, \cdot\rangle$ and norm $\|\cdot\|=\langle\cdot, \cdot\rangle^{1 / 2}$. It is not hard to verify that Dragomir's result (1.2) (with $\mathbb{F}=\mathbb{R}$ and $C, c>0$ ) can be obtained from Theorem 2.1 by setting

$$
\begin{gathered}
V_{1}=H, \quad V_{2}=X_{1}=X_{2}=\mathbb{R}, L_{1}=L_{2}=\mathbb{R}_{+}, \\
x \circ_{1} y=\langle x, y\rangle \text { for } x, y \in H, \quad \text { and } \alpha \circ_{2} \beta=\alpha \beta \text { for } \alpha, \beta \in \mathbb{R}, \\
\Phi(x)=\|x\| \text { for } x \in H, \quad \text { and } \Psi(\alpha)=|\alpha| \text { for } \alpha \in \mathbb{R} .
\end{gathered}
$$

In this case, (2.11) takes the form of inequality from [12, Lemma 1].

If $X_{i}$ is an algebra with unity $e_{i}$ and convex cone $L_{i} \subset X_{i}(i=1,2)$, then a linear map $\Psi: X_{1} \rightarrow X_{2}$ is said to be a unital positive map if $\Psi\left(e_{1}\right)=e_{2}$ and $\Psi L_{1} \subset L_{2}$.

ThEOREM 2.4. Under the assumptions before Theorem 2.1, let $V_{i}=X_{i}$ and let $\left(V_{i}, \circ_{i}\right)$ be algebra with unity $e_{i}(i=1,2)$.

Let $x \in V_{1}$ be such that

$$
0 \leq_{1}\left(x-c e_{1}\right) \circ_{1}\left(C e_{1}-x\right)
$$

for some scalars $C, c \in \mathbb{F}$ with $C c>0$ and $C+c>0$.

Assume that $\Psi: V_{1} \rightarrow V_{2}$ is a positive linear map (i.e., $\Psi L_{1} \subset L_{2}$ ) and $\Phi: V_{1} \rightarrow$ $V_{2}$ is a unital map (i.e., $\Phi\left(e_{1}\right)=e_{2}$ ) satisfying

$$
\Phi(x) \circ_{2} \Phi(x) \leq_{2} \Psi\left(x \circ_{1} x\right) \quad \text { and } \quad e_{2} \leq_{2} \Psi\left(e_{1}\right) .
$$

Then we have the inequality

$$
\Phi(x) \leq_{2} \frac{C+c}{2 \sqrt{C c}} \Psi(x) .
$$


Proof. Set $y=e_{1}$. Conditions (2.5)-(2.7) are fulfilled, because $\Psi$ is a positive linear map. Moreover, (2.15) gives (2.4). According to Theorem 2.1, we get (2.9) with $y=e_{1}$ and $\Phi(y)=e_{2}$. This proves (2.16).

Corollary 2.5. Under the assumptions of Theorem 2.4 for $V_{i}, X_{i}, L_{i}, \circ_{i}$ and $x$, suppose that for each $a \in L_{2}$ there exists unique vector $b=a^{1 / 2} \in L_{2}$ such that $b^{2}=b \circ_{2} b=a$.

Assume $\Psi: V_{1} \rightarrow V_{2}$ is a unital positive map. If (2.14) is met then we have the inequality

$$
\left[\Psi\left(x^{2}\right)\right]^{1 / 2} \leq_{2} \frac{C+c}{2 \sqrt{C c}} \Psi(x) .
$$

Proof. Define

$$
\Phi(v)=\left[\Psi\left(v^{2}\right)\right]^{1 / 2} \text { for } v \in V_{1} .
$$

Then $\Phi$ is unital, since $\Psi$ is so. It follows from (2.18) that (2.15) holds. Now, by using (2.16), we get (2.17).

By $\mathbb{M}_{p}$ and $\mathbb{H}_{p}$ we denote the linear spaces, respectively, of $p \times p$ complex matrices, and of $p \times p$ Hermitian matrices. The Loewner cone of all $p \times p$ positive semidefinite matrices is denoted by $\mathbb{L}_{p}$. For matrices $A, B \in \mathbb{M}_{p}$ we write $B \leq A$ if $A-B \in \mathbb{L}_{p}$. The symbol $I_{p}$ stands for the $p \times p$ identity matrix.

Remind that a linear map $\Psi: \mathbb{M}_{n} \rightarrow \mathbb{M}_{k}$ is said to be a unital positive map if $\Psi\left(I_{n}\right)=I_{k}$ and $\Psi \mathbb{L}_{n} \subset \mathbb{L}_{k}$ (see $\left.[4,14]\right)$. It is known that

$$
[\Psi(A)]^{2} \leq \Psi\left(A^{2}\right) \text { for } A \in \mathbb{L}_{n}
$$

(Kadison's inequality; see [1], [4, p. 2], [8]).

REMARK 2.6. (a) In the matrix setting, (2.17) reduces to a result of Ando [1]. Cf. also [6, Corollaries 2.5 and 2.9] and [17, Corollary 2.6, part (ii), $p=2]$.

(b) Inequality (2.17) generalizes a result of Liu and Neudecker [15, Proposition 5] (see also [6, Lemma 1.1]):

$$
\left(U^{*} X^{2} U\right)^{1 / 2} \leq \frac{M+m}{2 \sqrt{M m}} U^{*} X U,
$$

where $U$ is an $n \times k$ matrix such that $U^{*} U=I_{k}$, and $X$ is an $n \times n$ positive definite matrix satisfying

$$
0<m \leq \lambda_{j}(X) \leq M, j=1, \ldots, n, \quad \text { for some scalars } m, M .
$$


To see this, consider

$$
V_{1}=X_{1}=\mathbb{M}_{n}, \quad V_{2}=X_{2}=\mathbb{M}_{k}, \quad L_{1}=\mathbb{L}_{n}, \quad L_{2}=\mathbb{L}_{k},
$$

with the usual matrix multiplication, and

$$
\Psi(A)=U^{*} A U \quad \text { for } A \in \mathbb{M}_{n},
$$

where $U$ is an $n \times k$ matrix such that $U^{*} U=I_{k}$.

We now interpret Theorem 2.1 in the framework of $C^{*}$-algebras $V_{i}, i=1,2$, and unital positive maps. Here, for given $x, y \in V_{i}, y \leq x$ means $x-y=a^{*} a$ for some $a \in V_{i}$.

TheOREm 2.7. For $i=1,2$, let $V_{i}=X_{i}$ be a $C^{*}$-algebra with unity $e_{i}$ and convex cone $L_{i}=\left\{a^{*} a: a \in V_{i}\right\}$ of all nonnegative elements of $V_{i}$.

Let $x, y \in V_{1}$ be two elements such that $x^{*} y=y^{*} x$ and

$$
(x-c y)^{*}(C y-x) \geq 0 \text { for some positive scalars } C, c .
$$

Assume that $\Psi: V_{1} \rightarrow V_{2}$ is a unital positive map.

(I). If

$$
(\Psi(v))^{*} \Psi(v) \leq \Psi\left(v^{*} v\right) \quad \text { for } v \in\{x, y\}
$$

then we have the inequality

$$
\frac{1}{2}\left[(\Psi(x))^{*} \Psi(y)+(\Psi(y))^{*} \Psi(x)\right] \leq \frac{C+c}{2 \sqrt{C c}} \Psi\left(x^{*} y\right) .
$$

If, in addition, $\Psi(x)$ and $\Psi(y)$ are two commuting self-adjoint elements of $V_{2}$, then (2.24) becomes

$$
\Psi(x) \Psi(y) \leq \frac{C+c}{2 \sqrt{C c}} \Psi\left(x^{*} y\right)
$$

(II). We have the inequality

$$
\begin{array}{r}
\frac{1}{2}\left(\left[\Psi\left(x^{*} x\right)\right]^{1 / 2}\left[\Psi\left(y^{*} y\right)\right]^{1 / 2}+\left[\Psi\left(y^{*} y\right)\right]^{1 / 2}\left[\Psi\left(x^{*} x\right)\right]^{1 / 2}\right) \\
\leq \frac{C+c}{2 \sqrt{C c}} \Psi\left(x^{*} y\right) .
\end{array}
$$

If, in addition, $\left[\Psi\left(x^{*} x\right)\right]^{1 / 2}$ and $\left[\Psi\left(y^{*} y\right)\right]^{1 / 2}$ are two commuting elements of $V_{2}$, then we have the inequality

$$
\left[\Psi\left(x^{*} x\right)\right]^{1 / 2}\left[\Psi\left(y^{*} y\right)\right]^{1 / 2} \leq \frac{C+c}{2 \sqrt{C c}} \Psi\left(x^{*} y\right) .
$$


Proof. Put

$$
u \circ_{i} v=u^{*} v \text { for } u, v \in V_{i}, i=1,2 .
$$

Then $\circ_{i}$ is bilinear over $\mathbb{F}=\mathbb{R}$, and (2.1) is satisfied. Since $\Psi$ is a unital positive map, conditions (2.5)-(2.7) are fulfilled.

(I). Take $\Phi=\Psi$. Then (2.4) is met by (2.23). In consequence, by Theorem 2.1, inequalities (2.8) and (2.9) hold with $\Phi=\Psi$. Therefore (2.24) and (2.25) are valid.

(II). Choose $\Phi(v)=\left[\Psi\left(v^{*} v\right)\right]^{1 / 2}$ for $v \in V_{1}$. Then (2.4) holds automatically, and (2.26) and (2.27) follow directly from (2.8) and (2.9), respectively. $\square$

In the matrix setting if $\Phi=\Psi$ is a unital positive map, then condition (2.23) of Theorem 2.7 reduces to Kadison's inequality (2.19). In general, $\Psi$ and $\Phi$ need not be linear maps (see Remark 2.3).

We now discuss inequalities (2.14) and (2.22) which are crucial conditions for Theorems 2.4 and 2.7, respectively, to hold.

Lemma 2.8. Let $V_{1}$ be a $C^{*}$-algebra with unity $e_{1}$ and convex cone $L_{1}=\left\{a^{*} a\right.$ : $\left.a \in V_{1}\right\}$. Suppose that for each hermitian element $x \in V_{1}$ there exist real scalars $\lambda_{j}=\lambda_{j, x}$ and nonzero hermitian elements $a_{j}=a_{j, x} \in L_{1} j=1, \ldots, n$, such that

(i) $x=\lambda_{1} a_{1}+\ldots+\lambda_{n} a_{n}$,

(ii) $e_{1}=a_{1}+\ldots+a_{n}$,

(iii) $a_{j} a_{l}=a_{j}$ if $j=l$, and $a_{j} a_{l}=0$ if $j \neq l$,

(iv) $x \in L_{1}$ implies $\lambda_{1}, \ldots, \lambda_{n} \geq 0$.

Let $c, C \in \mathbb{R}$ and let $x, y \in V_{1}$ be two commuting hermitian elements with invertible $y$.

Consider conditions

$$
\begin{gathered}
c e_{1} \leq x y^{-1} \leq C e_{1}, \\
c \leq \lambda_{j, x y^{-1}} \leq C \quad \text { for } j=1, \ldots, n, \\
\left(x y^{-1}-c e_{1}\right)\left(C e_{1}-x y^{-1}\right) \geq 0, \\
(x-c y)(C y-x) \geq 0 .
\end{gathered}
$$

Then $(2.28) \Rightarrow(2.29) \Rightarrow(2.30) \Rightarrow(2.31)$. 
Proof. By (i) and (ii) applied to hermitian element $x y^{-1}$ we have

$$
\begin{gathered}
x y^{-1}-c e_{1}=\left(\lambda_{1}-c\right) a_{1}+\ldots+\left(\lambda_{n}-c\right) a_{n}, \\
C e_{1}-x y^{-1}=\left(C-\lambda_{1}\right) a_{1}+\ldots+\left(C-\lambda_{n}\right) a_{n} .
\end{gathered}
$$

If (2.28) holds, then $x y^{-1}-c e_{1} \in L_{1}$ and $C e_{1}-x y^{-1} \in L_{1}$. So, using (iv) and (2.32)-(2.33), we obtain

$$
\lambda_{j}-c \geq 0 \text { and } C-\lambda_{j} \geq 0 \text { for } j=1, \ldots, n,
$$

where $\lambda_{j}=\lambda_{j, x y^{-1}}$. This gives (2.29).

On the other hand, by (2.32)-(2.33) and (iii), we have

$$
\left(x y^{-1}-c e_{1}\right)\left(C e_{1}-x y^{-1}\right)=\left(\lambda_{1}-c\right)\left(C-\lambda_{1}\right) a_{1}+\ldots+\left(\lambda_{n}-c\right)\left(C-\lambda_{n}\right) a_{n} .
$$

In consequence, $(2.29)$ forces $(2.30)$ by $a_{j} \in L_{1}, j=1, \ldots, n$.

To see the implication $(2.30) \Rightarrow(2.31)$, it is sufficient to pre- and post-multiply (2.30) by $y^{*}=y$, and use the commutativity of $x$ and $y$. $\square$

Clearly, employing Lemma 2.8 for $y=e_{1}$, we obtain the implications

$$
c e_{1} \leq x \leq C e_{1} \Rightarrow c \leq \lambda_{j}(x) \leq C \Rightarrow 0 \leq\left(x-c e_{1}\right)\left(C e_{1}-x\right) .
$$

Lemma 2.8 gives possibility to produce Kantorovich type inequalities with various variants of assumptions on $x$ and $y$ (see [7, Theorems 2.1 and 2.4, Corollaries 2.2 and $2.3])$.

We now return to Theorem 2.7 and inequality (2.27).

Corollary 2.9. For $i=1,2$, let $V_{i}, X_{i}, L_{i}$ and $e_{i}$ be as in Theorem 2.7.

Let $x \in L_{1}$ be an invertible element such that

$$
\left(x-c e_{1}\right)\left(C e_{1}-x\right) \geq 0 \text { for some positive scalars } C, c .
$$

Assume that $\Psi: V_{1} \rightarrow V_{2}$ is a unital positive map. For any integer $p$, if $\Psi\left(x^{\frac{p+1}{2}}\right)$ and $\Psi\left(x^{\frac{p-1}{2}}\right)$ are two commuting elements of $V_{2}$, then we have the inequality

$$
\left[\Psi\left(x^{p+1}\right)\right]^{1 / 2}\left[\Psi\left(x^{p-1}\right)\right]^{1 / 2} \leq \frac{C+c}{2 \sqrt{C c}} \Psi\left(x^{p}\right) .
$$

Proof. It follows from Lemma 2.8 that (2.35) implies

$$
\left(x^{\frac{p+1}{2}}-c x^{\frac{p-1}{2}}\right)\left(C x^{\frac{p-1}{2}}-x^{\frac{p+1}{2}}\right) \geq 0 .
$$


That is (2.22) holds for $x^{\frac{p+1}{2}}$ and $x^{\frac{p-1}{2}}$. Applying (2.27), we obtain (2.36). $\mathrm{\square}$

ExAmPLE 2.10. The Kantorovich inequality (1.1) can be derived from Corollary 2.9 applied to the map

$$
\Psi(A)=z^{*} A z \quad \text { for } A \in \mathbb{M}_{n}
$$

where $z \in \mathbb{C}^{n}$ with $z^{*} z=1$. Indeed, $\Psi$ is a unital positive map from $\mathbb{M}_{n}$ to $\mathbb{C}$. Here

$$
V_{1}=X_{1}=\mathbb{M}_{n}, \quad L_{1}=\mathbb{L}_{n}, \quad V_{2}=X_{2}=\mathbb{C}, \quad L_{2}=\mathbb{R}_{+} .
$$

For $A>0$, let $0<c<C$ be scalars such that the spectrum of $A$ lies in the interval $[c, C]$. Then (2.36) with $x=A$ and $p=0$ becomes (1.1).

In a similar way, from (2.36) one can obtain the Schopf's inequality [20, p. 31]:

$$
z^{*} A^{p+1} z \cdot z^{*} A^{p-1} z \leq \frac{\left(\lambda_{1}+\lambda_{n}\right)^{2}}{4 \lambda_{1} \lambda_{n}}\left(z^{*} A^{p} z\right)^{2},
$$

where $p$ is an integer, and $\lambda_{1}$ and $\lambda_{n}$ are the largest and smallest eigenvalues of an $n \times n$ positive definite matrix $A$.

In the proof of Theorem 2.1, a key fact leading to (2.8) and (2.9) is inequality (2.10). (2.10) is a consequence of the bilinearity of the operation $\circ_{1}$. So, in order to get (2.9), it is possible to use (2.10) instead of the bilinearity of $\circ_{1}$. In fact, in the literature there are inequalities of types (2.10) and (2.9) with non-bilinear $\circ_{1}$.

ExAmPLE 2.11. Consider the following spaces and cones

$$
V_{1}=X_{1}=\mathbb{M}_{n}, \quad L_{1}=\mathbb{L}_{n}, \quad V_{2}=X_{2}=\mathbb{R}, \quad L_{2}=\mathbb{R}_{+} .
$$

Define maps as follows

$$
\Phi(A)=\left(z^{*} A z\right)^{1 / 2} \text { for } A \in \mathcal{A}=\mathbb{L}_{n}
$$

and

$$
\Psi(A)=z^{*} A z \quad \text { for } A \in \mathcal{B}=\mathbb{L}_{n}
$$

where $z \in \mathbb{C}^{n}$ with $z^{*} z=1$.

Take $\circ_{2}$ to be the usual multiplication on $\mathbb{R}$. Let $\circ_{1}$ be the binary operation of geometric mean [21, p. 689]:

$$
A \circ_{1} B=G(A, B)=A^{1 / 2}\left(A^{-1 / 2} B A^{-1 / 2}\right)^{1 / 2} A^{1 / 2} \quad \text { for } 0<A, B \in \mathbb{L}_{n} \text {. }
$$


With the aid of the version of Theorem 2.1 based on (2.10), we shall show how to obtain the inequality [21, Theorem 2.2]:

$$
\left(z^{*} A z\right)^{1 / 2}\left(z^{*} B z\right)^{1 / 2} \leq \frac{C+c}{2 \sqrt{C c}} z^{*} G(A, B) z
$$

for $0<A, B \in \mathbb{L}_{n}$ with $0<c I_{n} \leq A, B \leq C I_{n}$ and $0<c<C$.

To do this, we use the result $[13,21]$ :

$$
\frac{1}{2}(A+B) \leq \frac{C+c}{2 \sqrt{C c}} G(A, B)
$$

for $0<A, B \in \mathbb{L}_{n}$ with $0<c I_{n} \leq A, B \leq C I_{n}$ and $0<c<C$. Because $G(A, \alpha B)=$ $\alpha^{1 / 2} G(A, B)$ for $\alpha>0$ [21, p. 689], substituting $C c B$ instead of $B$ leads to

$$
A+C c B \leq(C+c) G(A, B),
$$

which is of the form (2.10).

Furthermore, $G(A, B)=G(B, A)[21$, p. 689]. Clearly, conditions (2.5)-(2.7) are satisfied. Since $G(A, A)=A[21$, p. 689], it is readily seen that (2.4) is met.

By the discussion before this example, we get (2.9). It is not hard to check that (2.9), with $\Phi$ and $\Psi$ defined by (2.37) and (2.38), can be rewritten as (2.39).

Acknowledgment. The author thanks an anonymous referee for his/her valuable comments.

\section{REFERENCES}

[1] T. Ando. Quasi-orders on the positive cone of a $C^{*}$-algebra. Linear and Multilinear Algebra, 41:81-94, 1996.

[2] J. K. Baksalary and S. Puntanen. Generalized matrix versions of the Cauchy-Schwarz and Kantorovich inequalities. Aequationes Mathematicae, 41:103-110, 1991.

[3] R. Bhatia. Matrix Analysis. Springer-Verlag, New York, 1997.

[4] R. Bhatia and C. Davis. A key inequality for functions of matrices. Linear Algebra and its Applications, 323:1-5, 2001.

[5] J.-C. Bourin. Matrix versions of some classical inequalities. Linear Algebra and its Applications, 416:890-907, 2006.

[6] J.-C. Bourin. Symmetric norms and reverse inequalities to Davis and Hansen-Pedersen characterizations of operator convexity. Mathematical Inequalities \& Applications, 9:33-42, 2006.

[7] J.-C. Bourin. Reverse rearrangement inequalities via matrix technics. Journal of Inequalities in Pure and Applied Mathematics, 7 (2) (2006) Article 43. [Online: http://jipam.vu.edu.au/article.php?sid=660]

[8] M.D. Choi. A Schwarz inequality for positive linear maps on $C^{*}$-algebras. Illinois Journal of Mathematics, 18:565-574, 1974. 
[9] S.S. Dragomir. A generalization of Grüss' inequality in inner product spaces and applications. Journal of Mathematical Analysis and Applications, 237:74-82, 1999.

[10] S.S. Dragomir. Some companions of the Grüss inequality in inner product spaces. Journal of Inequalities in Pure and Applied Mathematics, 4 (5) (2003) Article 87. [Online: http://jipam.vu.edu.au/article.php?sid=328]

[11] S.S. Dragomir. Reverses of Schwarz, triangle and Bessel inequalities in inner product spaces. Journal of Inequalities in Pure and Applied Mathematics, 5 (3) (2004) Article 76. [Online: http://jipam.vu.edu.au/article.php?sid=368] .

[12] N. Elezović, L. Marangunić and J. Pečarić. Unified treatment of complemented Schwarz and Grüss inequalities in inner product spaces. Mathematical Inequalities \& Applications, 8:223-231, 2005.

[13] M. Fujii, S. Izumino, R. Nakamoto and Y. Seo. Operator inequalities related to Cauchy-Schwarz and Hölder-McCarthy inequalities. Nihonkai Mathematical Journal, 8:117-122, 1997.

[14] C.-K. Li and R. Mathias. Matrix inequalities involving a positive linear map. Linear and Multilinear Algebra, 41:221-231, 1996.

[15] S. Liu and H. Neudecker. Several matrix Kantorovich-type inequalities. Journal of Mathematical Analysis and Applications, 197:23-26, 1996.

[16] A. W. Marshall and I. Olkin. Matrix versions of Cauchy and Kantorovich inequalities. Aequationes Mathematicae, 40:89-93, 1990.

[17] J. Mićić, J. Pečarić and Y. Seo. Complementary inequalities to inequalities of Jensen and Ando based on the Mond-Pečarić method. Linear Algebra and its Applications, 318:87-107, 2000.

[18] B. Mond and J. Pečarić. Matrix inequalities for convex functions. Journal of Mathematical Analysis and Applications, 209:147-153, 1997.

[19] M. Niezgoda. Translation invariant maps and applications. Journal of Mathematical Analysis and Applications, 354:111-124, 2009.

[20] G.S. Watson, G. Alpargu and G.P.H. Styan. Some comments on six inequalities associated with the inefficiency of ordinary least squares with one regressor. Linear Algebra and its Applications, 264:13-54, 1997.

[21] T. Yamazaki. An extension of Kantorovich inequality to $n$-operators via the geometric mean by Ando-Li-Mathias. Linear Algebra and its Applications, 416:688-695, 2006. 